This item was submitted to Loughborough's Research Repository by the author.

Items in Figshare are protected by copyright, with all rights reserved, unless otherwise indicated.

\title{
Brachial artery characteristics and micro-vascular filtration capacity in rock climbers
}

PLEASE CITE THE PUBLISHED VERSION

http://dx.doi.org/10.1080/17461391.2014.940560

\section{PUBLISHER}

(C) Taylor \& Francis Group

\section{VERSION}

AM (Accepted Manuscript)

\section{PUBLISHER STATEMENT}

This work is made available according to the conditions of the Creative Commons Attribution-NonCommercialNoDerivatives 4.0 International (CC BY-NC-ND 4.0) licence. Full details of this licence are available at: https://creativecommons.org/licenses/by-nc-nd/4.0/

\section{LICENCE}

CC BY-NC-ND 4.0

\section{REPOSITORY RECORD}

Thompson, Emilia, Luke Farrow, Julie E.A. Hunt, Mark P. Lewis, and Richard A. Ferguson. 2019. "Brachial Artery Characteristics and Micro-vascular Filtration Capacity in Rock Climbers". figshare.

https://hdl.handle.net/2134/19298. 


\title{
Brachial artery characteristics and micro-vascular filtration capacity in rock climbers
}

\begin{abstract}
Rock climbers perform repeated isometric forearm muscle contractions subjecting the vasculature to repeated ischaemia and distorted haemodynamic signals. This study investigated forearm vascular characteristics in rock climbers compared to healthy untrained controls. 8 climbers (CLIMB) (BMI; 22.3, $s=2.0 \mathrm{~kg} / \mathrm{m}^{2}$, isometric handgrip strength; 46, $s=$ $8 \mathrm{~kg}$ ) were compared against 8 untrained controls (CON) (BMI; 22.8, s=2.6 kg/m², isometric handgrip strength; 37, s = $9 \mathrm{~kg}$ ). Brachial artery diameter and blood flow were measured, using Doppler ultrasound, at rest and following 5-mins ischaemia (peak diameter) and ischaemic exercise (maximal dilation) to calculate flow mediated dilation (FMD) and dilatory capacity (DC). Capillary filtration capacity was assessed using venous occlusion plethysmography. Resting (4.30, $s=0.26$ vs. 3.79, $s=0.39 \mathrm{~mm})$, peak $(4.67, s=0.31$ vs. $4.12, s=0.45 \mathrm{~mm})$ and maximal $(5.14, s=0.42$ vs. $4.35, \mathrm{~s}=0.47 \mathrm{~mm})$ diameters were greater $(P<0.05)$ in CLIMB than CON, respectively, despite no difference in FMD $(9.2, \mathrm{~s}=2.6$ vs. 8.7, $\mathrm{s}=2.9 \%$ \%. Peak reactive hyperaemic blood flow (1136, $s=504$ vs. $651, \mathrm{~s}=221 \mathrm{ml} / \mathrm{min}$ ) and capillary filtration capacity $\left(3.8, s=0.9\right.$ vs. $5.2, s=0.7 \mathrm{ml} \cdot \mathrm{min}^{-1} \cdot \mathrm{mmHg}^{-1} \cdot 100 \mathrm{ml}$ tissue $\mathrm{e}^{-1}$ $\left.\mathrm{x} 10^{-3}\right)$ were greater $(P<0.05)$ in CLIMB compared to CON, respectively. In conclusion, rock climbers exhibit structural vascular adaptation compared to untrained control participants but have similar vascular function. This may contribute to the enhanced ability of climbers to perform repeated isometric contractions.
\end{abstract}

Key words: ischaemic exercise; blood flow; forearm; remodelling 


\section{Introduction}

It is now evident that well-trained athletes undergo significant adaptations to the peripheral vasculature. Structural adaptations, including a relatively greater diameter of the conduit arteries have been observed (Huonker, Schmid, Schmid-Truckäss, Grathwohl and Keul, 2003; Rowley, Dawson, Birk, Cable, George, Whyte, Thijssen and Green, 2011a; Rowley, Dawson, Hopman, George, Whyte, Thijssen and Green, 2011b) despite a lack of a functional improvement or in some cases a functional decrease (Petersen et al., 2006; Rowley et al., 2011b). This phenomenon has recently been called the 'athletes paradox' (Green, Spence, Rowley, Thijssen and Naylor, 2012).

Rock-climbers typically exhibit small statures and low body fatness with high strength to weight ratios (Watts, Martin and Durtschi, 1993). Despite previous anthropometric profiling of this group of athletes, the specific characteristics of the peripheral vasculature remain relatively poorly understood. Ferguson and Brown (1997) identified a higher peak vascular conductance and forearm vasodilatory capacity in climbers allowing for greater functional hyperaemia compared with non-climbers, suggesting adaptation of the resistance vessels. Rock climbing requires continuous work at approximately 50\% of maximal aerobic capacity which increases with levels of climbing difficulty (Sheel, 2004). It involves repeated isometric forearm muscle contractions (Macleod, Sutherland, Buntin, Whitaker, Aitchison, Watt, Bradley and Grant, 2007) and whilst the precise level of forces produced during ascents are unknown the percentage of forearm maximal voluntary contraction at which climbers must work vary considerably, especially given the occasional dependence on lower limbs for upward propulsion (Sheel, Seddon, Knight, McKenzie and Warburton, 2003), where approximately $57 \%$ of vertical forces are applied to the feet in the standard vertical position (Noẻ, Quaine and Martin, 2001). Although intramuscular pressure may vary between muscle groups and individuals, blood flow is insufficient to maintain homeostasis at intensities 
greater than 10\% MVC (Sjøgaard, Savard and Juel, 1988). Thus, although no specific data exists surrounding the proportion of MVC utilised during climb, it is fair to speculate that the exercising muscle and related vasculature is subjected to regular periods of ischaemia (Sadamoto, Bonde-Petersen and Suzuki, 1983).

Although shear stress is suggested to be the predominant stimulus for conduit artery adaptation with training (Laughlin, Newcomer and Bender, 2008; Newcomer, Thijssen and Green, 2011; Tinken et al., 2010), the episodic reductions in oxygen availability as a result of muscle ischaemia may also play a role via AMPK mediated (Chen et al, 2009) eNOS activation (Rudic et al 1998) as well as the role played by VEGF and HIF-1 $\alpha$ in angiogenesis (Egginton, 2009). One model of exercise that has allowed examination of such vascular adaptations and in some respect may reflect the conditions encountered in the active muscles and vasculature during rock climbing, is training under blood flow restricted (BFR) conditions which has been shown to increase conduit artery diameter (Hunt, Walton and Ferguson, 2012; Hunt, Galea, Tufft, Bunce and Ferguson, 2013). Adaptations are also evident through the vascular tree and peripheral resistance vessels also exhibit remodelling with training (Ferguson and Brown, 1997; Martin, 1987; Sinoway, Musch, Minotti and Zelis, 1986; Snell, Martin, Buckey and Bloomqvist, 1987). Further downstream, adaptation of the microvasculature has been observed with short term training in a blood flow restricted state providing a stimulus for a greater microvascular filtration capacity in calf muscles (Evans, Vance and Brown 2010).

Given the paucity of data in this athletic population the aim of this study was to determine vascular adaptations in rock climbers, from the level of large conduit arteries to the microvascular capillary network. In accordance with the 'athletes paradox' theory (Green et al., 2012) it was hypothesised that rock climbers would exhibit an enlarged artery diameter, suggestive of arterial remodelling, but there would be an absence of any functional 
adaptations. It was also hypothesised that rock climbers would exhibit an enhanced capillary filtration capacity, reflective of a greater capillary density (Brown, Jeal, Bryant and Gamble, 2001). 


\section{Methods}

Participants

A total of 16 healthy males volunteered to take part in the study. 8 were rock climbers (CLIMB) recruited via advertisement at local climbing centres, and 8 age-matched individuals recruited from the local university population as control participants (CON) (Table 1) Post-hoc power analysis revealed a minimum of 96\% statistical power (at an $\alpha$ level of 0.05) for all brachial artery measurements. Climbers had a minimum of 3 years climbing experience and were able to climb at a grade of F6a (5.10a Yosemite Decimal System or equivalent) or above. This level equates to the minimum in the 'extremely severe' category of climbs. Control participants were habitually physically active but none performed resistance exercise training or racket sports. All participants completed a health screening questionnaire and were fully informed of the purposes, risks and discomforts associated with the experiment before providing written, informed consent. Participants with known cardiovascular, respiratory, haematological or metabolic disease and those currently taking any medication were excluded from the study. This study conformed to local guidelines and the Declaration of Helsinki and was approved by Loughborough University Ethics Advisory Committee.

\section{Experimental protocol}

After an overnight fast, participants reported to the laboratory (at approximately 8am) having refrained from strenuous physical activity for 24 hours, and abstaining from the consumption of alcohol, caffeine, tobacco and vitamin supplementation for 12 hours. Following an initial familiarisation to the protocol and equipment the participant rested for a minimum of 20 minutes in a supine position in a quiet temperature-controlled $\left(24, s=1^{\circ} \mathrm{C}\right)$ room. Initially, Doppler ultrasound was used to assess brachial artery function (flow mediated dilation; 
FMD), structure (maximum dilatory capacity; DC) and blood flow. All measures were taken on the right arm, which was the dominant arm in all participants. After 40 a minute break from testing the participant returned to a supine position for a further 20 min before resting forearm blood flow and capillary filtration capacity was determined using venous occlusion plethysmography on the same arm. Following vascular measures, forearm strength and anthropometry measures were obtained.

\section{Brachial artery assessments}

Doppler ultrasound imaging was performed by the same sonographer using a Toshiba Powervision 6000 with a multi-frequency linear array transducer (7-11 MHz). Participants lay supine with the imaged arm extended and immobilised at an angle approximately $80^{\circ}$ from the torso. A $90^{\circ} \mathrm{B}$-mode image of the brachial artery at $3 \mathrm{~cm}$ depth was obtained $>3 \mathrm{~cm}$ proximal to the olecranon process. In duplex mode the sample volume was centralised within the artery and the ultrasound beam aligned with direction of flow (insonation angle $\leq 69^{\circ}$ ). Once a satisfactory image was obtained, the probe was held in a constant position for the duration of the test. A vascular ECG gating module (Medical Imaging Applications, LLC, Coralville, Iowa, USA) triggered acquisition of the ultrasound images on the R-wave pulse of an ECG signal. Sequential end-diastolic images were stored from on-line image digitization.

Flow mediated dilation (FMD). Resting brachial diameter and blood flow were recorded for 30 cardiac cycles. For the ischaemic FMD stimulus a pneumatic cuff (E20 Rapid cuff inflator and AG101 Cuff Inflator Air Source, Hokanson, WA, USA) was placed immediately distal to the olecranon process and inflated to $200 \mathrm{mmHg}$. Occlusion was maintained for $5 \mathrm{~min}$ before rapid cuff deflation. Recording of real-time duplex imaging was resumed $10 \mathrm{sec}$ before deflation and continued for $\sim 3$ min post-deflation, capturing the transient changes in flow and diameter over a total of 200 cardiac cycles. 
Dilatory capacity (DC.) Following a minimum of 10 minutes supine rest, resting brachial diameter and blood flow were re-measured before commencing the DC protocol Subsequent analysis confirmed the resting status of these values (i.e. $0.6 \%$ and $5.3 \%$ of initial resting values for diameter and blood flow, respectively). For the ischaemic exercise DC stimulus, the pneumatic cuff was positioned on the upper arm proximal to the ultrasound probe. The cuff was inflated to $200 \mathrm{mmHg}$ with occlusion maintained for 5 min. During the middle 3 min, rhythmic isometric handgrip exercise was performed with a handgrip dynamometer. Real-time duplex imaging was performed as described for FMD.

Ultrasound Data Analysis. Brachial artery diameter and flow velocity were analysed with a custom-designed, edge detection and wall tracking software (Vascular Research Tools 5, Medical Imaging Applications, LLC, Coralville, Iowa, USA). Media-to-media diastolic diameter was measured within a specified region of interest on B-mode images. The Doppler flow velocity spectrum was traced and time average mean velocity (TAMV) $(\mathrm{cm} / \mathrm{s})$ computed. Synchronised diameter and velocity data, sampled at $20 \mathrm{~Hz}$, enabled calculation of blood flow and shear rate. Resting diastolic diameter (mm) was averaged over 30 cardiac cycles. The dilatory response to FMD and DC protocols was determined from smoothed data (moving average across 3 cardiac cycles) and peak and maximal diameter defined, respectively. FMD and DC are presented as the absolute ( $\mathrm{mm})$ and relative (\%) change in post-stimulus diameter; (maximum post stimulus diameter - baseline diameter)/baseline diameter. Time to peak diameter (s) was calculated from the point of cuff deflation to the maximum post-deflation diameter. Blood flow $(\mathrm{ml} / \mathrm{min})$ was calculated as (TAMV $\left.\mathrm{x} \pi \mathrm{r}^{2}\right) \mathrm{x}$ 60 , where $r$ is the radius of the brachial artery lumen. Resting blood flow was averaged over 30 cardiac cycles. Peak blood flow was recorded as the highest value (across a single cardiac cycle) following cuff deflation. Shear rate was derived from Poiseuillies law and calculated accordingly as (4 x TAMV) / diameter. The accumulated shear stimulus contributing to the 
FMD response was defined as the area under the shear rate curve $\left(\mathrm{SR}_{\mathrm{AUC}}\right)$ calculated for data from the time of cuff deflation to the point of peak dilation for each individual. The day-today reproducibility of brachial artery measurements was; diameter (0.7\%), blood flow (9.1\%), FMD\% (7.0\%) and DC\% (3.8\%) respectively.

\section{Capillary filtration capacity}

Venous occlusion strain-gauge plethysmography (Hokanson, Bellvue, WA, USA) was used to determine forearm capillary filtration capacity. With the forearm supported above the level of the heart and the subject lying supine, a pneumatic cuff (E20 Rapid cuff inflator and AG101 Cuff Inflator Air Source, Hokanson, WA, USA) was positioned proximal to the olecranon process. A mercury strain gauge was placed around the widest portion of the forearm and attached to a dual channel plethysmograph (EC6 Plethysmograph, Hokanson, Bellvue, WA, USA). After 20 min supine rest, 4-min cumulative pressure increments of 8 mmHg (not exceeding diastolic pressure) were applied to the cuff, lasting a total of 32 minutes after which point the cuff pressure was released and strain gauge removed. Change in forearm volume was detected throughout through strain gauge signals sampled on line at $100 \mathrm{~Hz}$ (Powerlab, ADInstruments, NSA, Australia) and measured using Chart version 5 software (ADInstruments, NSW, Australia). Increments beyond isovolumetric venous pressure induced a two-phase calf volume response comprising of; 1) an initial rapid phase attributed to venous filling and 2) a subsequent slower phase attributed to fluid filtration (Gamble et al. 1993). The fluid filtration rate $\left(\mathrm{J}_{\mathrm{v}}\right)$ was measured from the slope of volume change during the $2-4^{\text {th }}$ min of each pressure step. This filtration rate was plotted against cuff pressure and the slope of the linear relationship defined the filtration capacity $\left(\mathrm{K}_{\mathrm{f}} ; 10-3 \mathrm{x}\right.$ ml.min ${ }^{-1} \cdot \mathrm{mmHg}^{-1} \cdot 100 \mathrm{ml}$ tissue $\mathrm{e}^{-1}$.

\section{Forearm strength and anthropometry}


Maximum isometric handgrip strength was determined in the right arm using a handgrip dynamometer (Jamar, Sammons Preston Rolyan, Bolingbrook, IL, USA). The participants stood with the elbow flexed at $90^{\circ}$. Three maximal contractions were performed for $\sim 3$ seconds, with a rest interval of 3 minutes between each contraction. The maximum value obtained from the 3 contractions was recorded as the maximum isometric strength.

\section{Statistical analysis}

A Shapiro-Wilk test was used to confirm normal distribution and a Mauchley test of sphericity to verify homogeneity of variance. Independent $t$-tests were conducted to compare the differences between the two groups. Baseline and peak diameters were log transformed and a one-way analysis of covariance (ANCOVA) performed with the difference between transformed baseline and peak diameters used as the dependent variable and transformed baseline diameter the covariate. This accounted for the effect of differences in baseline artery diameter between groups on FMD\% (Atkinson and Batterham, 2013a). Means were subsequently antilogged and interpreted as FMD\%. This was also conducted for maximum diameters to scale DC\% values. All data are presented as mean, $s=$ standard deviation and significance was accepted at $P<0.05$. 


\section{Results}

There were no differences in anthropometrical measures between CLIMB and CON, and despite no significant difference in forearm circumference or maximum isometric handgrip strength, after correcting for body mass CLIMB exhibited a greater strength to body mass ratio compared to CON (Table 1).

\section{Brachial artery measures}

All data are shown in Table 2 . Resting diameter was $11.8 \%$ greater in CLIMB than CON. Blood flow and shear rate at rest were not different between groups. Peak diameter in response to the ischaemic stimulus was $11.7 \%$ greater in CLIMB than CON, however there was no difference in time to peak diameter. There were no differences in both relative (\%) and absolute (mm) FMD or shear stimulus $\left(\mathrm{SR}_{\mathrm{AUC}}\right)$ between groups. This lack of difference was still present following normalisation of FMD for shear. Following allometric scaling to account for baseline diameter, FMD $\%$ was $8.2, s=3.2 \%$ and $9.0, s=3.2 \%$ in CLIMB and CON, respectively with no difference between groups $(P=0.663)$.

Maximal diameter was $15.3 \%$ greater and DC $25.5 \%$ greater in CLIMB than CON, respectively (Table 1). Following allometric scaling, DC\% was 19.8, $s=4.9 \%$ and 13.7, $s=$ 5.5\% in CLIMB and CON, respectively with no difference between groups $(P=0.085)$.

\section{Capillary filtration capacity}

There was no difference in resting forearm blood flow. Capillary filtration capacity was 27.4\% greater in CLIMB compared to CON (Table 3). 


\section{Discussion}

The present study has demonstrated that rock climbers exhibit greater arterial diameters compared to untrained control participants. However, there was no difference in FMD between groups. Rock climbers also exhibited an enhanced capillary filtration capacity, suggestive of a greater capillary density. Taken together with the observations of resistance vessel adaptation (Ferguson and Brown, 1997), rock climbers evidently possess an enhanced vasculature at all levels of the arterial tree and microcirculation and offer a unique insight into the vascular adaptations with chronic training in repeated isometric ischaemic conditions.

The present observation of an enhanced brachial artery diameter in rock climbers supports the large body of evidence of a greater peripheral conduit artery size in well-trained populations. For example, chronically trained endurance athletes exhibit an overall increase in arterial size, specifically in the common femoral arteries of endurance athletes which are both structurally and functionally adapted compared with inactive individuals (Schmidt-Trucksäss et al., 2000). Rowley et al. (2011b) observed larger femoral artery diameters in runners and cyclists and in the playing arm of elite squash players. Larger vessels are also a feature in the upper-body of wheelchair athletes (Zeppilli et al., 1995). An enlargement of the arteries in trained individuals may contribute to an improvement in performance through an enhanced blood flow and oxygen delivery (Green et al., 2012; Welsch, Blalock, Credeur and Parish, 2013). Although it is appreciated that local blood flow is regulated by the resistance vessels we have previously demonstrated resistance vessel adaptation resulting in an enhanced limb blood flow capacity and functional hyperaemia in climbers (Ferguson and Brown, 1997). Moreover, there is a greater potential for enhanced oxygen extraction as demonstrated by the enhanced capillary filtration capacity.

The higher basal diameter observed in rock climbers may be due to altered sympathetic vasomotor tone, circulating hormones and local paracrine effects. A reduction in sympathetic 
activity in trained individuals (Lehmann, Schmid and Keul, 1984) may extrinsically alter basal vascular tone and stimulate vasodilation. Further, chronic exercise training may lower plasma concentrations of the vasoconstrictor hormones endothelin-1 (ET-1) (Maeda et al., 2009) and angiotensin II (Braith, Welsch, Feigenbaum, Kluess and Pepine, 1999), although a relationship between ET-1 and basal vascular tone is disputed (Thijssen et al., 2008). Additionally, the bioactivity of nitric oxide (NO), a potent stimulus for enlargement of vessels (Rudic et al., 1998) is greater with training (Green, Maiorana, O’Driscoll and Taylor, 2004; Kingwell, Sherrard, Jennings and Dart, 1997) and may present as a larger conduit vessel diameter due to its contribution to basal arterial tone (Vallance, Collier and Moncada, 1989). Despite this, the greater maximal diameter (stimulated by ischaemic exercise) in the present study suggests the presence of vascular remodelling (Naylor, Weisbrod, O’Driscoll and Green, 2005).

The key physiological stimulus proposed to be responsible for the remodelling of the conduit arteries is the repetitive shear stress produced during exercise (Langille and O’Donnell, 1986; Tinken et al., 2010; Green et al., 2012) which activates a number of signalling cascades within the endothelial cells resulting in an altered endothelial cell phenotype (Chien, 2007) and consequent remodelling. Independent of, or indeed in addition to the effects of shear stress, there may be contributions from other stimuli e.g. hypoxic/metabolic conditions via AMPK mediated (Chen et al, 2009) eNOS activation which has been shown to be obligatory for arterial remodelling (Rudic et al 1998). Additionally, lactate released by contracting muscle may up-regulate VEGF and its receptor VEGFR2 (Kumar, Viji, Kiran and Sudhakaran, 2007). Indeed, a strong correlation exists between lactate and exercise induced changes in VEGF protein (Takano et al., 2005). However, although VEGF leads to the upregulation of eNOS, the direct effect on arteriogenesis is currently undetermined (Prior, Yang and Terjung, 2004). 
The absence of any enhancement in FMD in climbers is perhaps not surprising and corresponds to the mounting evidence regarding the impact of exercise training on artery function. Although improved function has been observed with exercise training in patients with cardiovascular disease (Hambrecht et al., 2003), the same has not been observed in healthy athletic populations (Tinken, Thijssen, Black, Cable and Green, 2008; Rowley et al., 2011b). It is now apparent that an 'athlete paradox' exists, whereby despite the expectation of greater vascular function in highly trained individuals, this group exhibit functionally 'normal' arteries (Green et al., 2012). This lack of functional adaptation can be also explained by appreciating the time-course of artery adaptation to exercise training. Although the crosssectional nature of the present and other studies does not allow us to elucidate this, previous work using aerobic type exercise training has demonstrated that functional changes occur rapidly and are then superseded by structural changes with artery function returning to baseline (Tinken et al., 2008). We have also observed a similar time-course of adaptation in response to BFR exercise (Hunt et al, 2012; Hunt et al, 2013), a model of exercise that may reflect the conditions encountered in the active muscles and vasculature during rock climbing. Our results therefore appear to be in accordance with the 'athlete artery' paradigm, and suggest repeated isometric contractions results in structural adaptation of the conduit arteries, although we do appreciate that longitudinal data on the effect of this type of specific training is lacking and the impact of distinct forms of exercise, such as in weight- or powertrained individuals have not been specifically investigated (Green et al., 2012).

Given that artery function may be overestimated in those with smaller baseline diameter and conversely be underestimated in those with a larger baseline diameter (Atkinson and Batterham, 2013a), we employed a relatively novel allometric scaling approach to our data (Atkinson and Batterham, 2013b) to minimise the potential bias of FMD\% values. Due to the inverse relationship between baseline diameter and FMD\% (Celermajer et al., 1992) and the 
observed greater baseline diameter in climbers than controls in the present study, it was of interest to determine whether differences between groups, or lack thereof, were still apparent following allometric adjustments and hence removal of statistical bias. Although no difference was observed between groups following FMD\%, the differences between groups in DC\% lost significance after baseline diameter bias was removed. Structural differences in diameter between groups were nonetheless confirmed given the observed differences in resting, peak and maximal diameter.

Despite our observations of enhanced brachial artery diameters in rock climbers, the influence of body mass and forearm size must be taken into consideration (Hopkins et al., 2009). Firstly, whole body mass was not different between CLIMB and CON (Table 1). Importantly there were no initial correlations between whole body mass and artery diameters (Table 4). Subsequent scaling of artery diameters with body mass did reveal significant correlations (Table 4) and suggest that appropriate allometric scaling should be performed which, as predicted, produced size independent normalised data (Table 4). Hopkins et al (2009) also observed that anthropometrical segmental measures had an influence on artery diameter similar to whole body mass. Unfortunately, direct measures of forearm muscle mass were not taken in the present study. However, forearm circumference has previously been shown to correlate with muscle mass (Kallman, Plato and Tobin, 1990). Like whole body mass, forearm circumference was not different between CLIMB and CON (Table 1), although there was a tendency for a larger circumference in CLIMB. Subsequent analysis revealed no correlations between forearm circumference and artery diameters and neither when artery diameters were scaled for forearm circumference (Table 4). Therefore, our data does not necessarily support the notion of scaling or normalisation for brachial artery diameters in the present subject cohort, since segmental measures (albeit not completely optimal) did not reveal any size dependence. 
Adaptation of resistance vessels (peak reactive hyperaemia) was also observed in climbers compared with untrained control participants and is in line with previous observations of an enhanced post-occlusion reactive hyperaemic blood flow in rock climbers (Ferguson and Brown, 1997) and other athletic populations (Martin et al., 1987; Sinoway et al., 1986; Snell et al., 1987). The magnitude of post-occlusion blood flow is governed by resistance vessel cross-sectional area (Naylor, Fitzsimons, Cable, Thijssen and Green, 2005) and therefore influenced by vessel function and structure. It is possible that there is a greater responsiveness to metabolic and endothelial vasodilators during periods of ischaemia. It is also plausible that rock climbers exhibit a greater number of resistance vessels, allowing for augmented blood flow into the forearm in conjunction with attenuated outflow by virtue of decreased venous congestion. This could be brought about by an increased number of capillaries and/or venules in parallel (Egaña, Reilly and Green, 2010). Indeed, we have observed a greater capillary filtration capacity in climbers suggesting an increase in the muscle capillary density of these individuals (Brown et al., 2001). Exercise training has long been recognised as a potent stimulus for increasing muscle capillarity (Eggington, 2009). The addition of a hypoxic stimulus may stimulate capillary growth via the up-regulation of various growth factors, the likely principal mediator being VEGF (Eggington, 2009), As previously discussed, intermittent ischaemic training in rock climbers may up-regulate the expression of HIF-1 $\alpha$, which itself leads to an increase in VEGF expression and capillary growth (Hoppeler, 1999). Indeed, microvascular filtration capacity has previously been shown to be enhanced with short-term low-intensity exercise training with blood flow restriction (Evans et al., 2010).

In conclusion, this study has identified an enhanced peripheral vasculature in rock climbers compared to untrained control participants. Rock climbers exhibit greater arterial diameters compared to sedentary control participants despite no difference in FMD between groups. 
These results are in accordance with the ‘athlete’s artery’ paradigm (Green et al., 2012) of an enlarged arterial lumen despite being functionally 'normal'. Additionally, rock climbers exhibit an enhanced capillary filtration capacity. The present data, together with the observations of resistance vessel adaptations (Ferguson and Brown, 1997) clearly demonstrate an enhanced peripheral vasculature in rock climbers. An enhancement in resistance vessel and/or micro-vascular function and structure would subsequently enhance functional blood flow, hence substrate exchange and metabolite removal, critical in individual performance and longevity of the climb. These observations also offer a unique insight into vascular adaptation with chronic training in intermittent blood flow restricted conditions. Future studies are required to investigate the mechanisms responsible for such adaptations throughout the vascular tree, and the cellular and molecular mechanisms underpinning these changes. Future studies should also examine the acute haemodynamic responses to climbing specific exercise to fully establish whether the adaptations observed translate to functional changes in blood flow. 


\section{References}

Atkinson, G., \& Batterham, A. M. (2013a). Allometric scaling of diameter change in the original flow-mediated dilation protocol. Atherosclerosis, 226(2), 425-427.

Atkinson, G., \& Batterham, A. M. (2013b). The percentage flow-mediated dilation index: A large-sample investigation of its appropriateness, potential for bias and causal nexus in vascular medicine. Vascular Medicine, 0(0), 1-12

Braith, R., Welsch, M. A., Feigenbaum, M. S., Kluess, H. A., \& Pepine, C. J. (1999) Neuroendocrine activation in heart failure is modified by endurance exercise training. Journal of the American College of Cardiology, 34(4), 1170-1175.

Brown, M. D., Jeal, S., Bryant, J., \& Gamble, J. (2001). Modifications of microvascular filtration capacity in human limbs by training and electrical stimulation. Acta Physiologica Scandinavica, 173, 359-368.

Celermajer, D. S., Sorensen, K. E., Gooch, V. M., Sullivan, I. D., Lloyd, J. K., Deanfield, J. E., \& Spiegelhalter, D. J. (1992). Non-invasive detection of endothelial dysfunction in children and adults at risk of atherosclerosis. The Lancet, 340(8828), 1111-1115.

Chen, Z., Peng, I. C., Sun, W., Su, M. I., Hsu, P. H., Fu, Y., Zhu, Y., DeFea, K., Pan, S., Tsai, M., \& Shyy, J. Y.(2009). AMP-activated protein kinase functionally phosphorylates endothelial nitric oxide synthase Ser633. Circulation research, 104(4), 496-505.

Chien, S. (2007) Mechanotransduction and endothelial cell homeostasis: the wisdom of the cell. Am. J. Physiol. Heart Circ. Physiol. 292, 1209-1224

Egaña, M., Reilly, H., \& Green, S. (2010). Effect of elastic-band-based resistance training on leg blood flow in elderly women. Applied Physiology, Nutrition, and Metabolsim, 35(6), 763-72. 
Egginton, S. (2009). Invited review: activity-induced angiogenesis. European Journal of Physiology, 457, 963-977.

Evans, C., Vance, S., \& Brown, M. (2010). Short term resistance training with blood flow restriction enhances microvascular filtration capacity of human calf muscles. Journal of Sports Sciences, 28(9), 999-1007.

Ferguson, R. A., \& Brown, M. D. (1997). Arterial blood pressure and forearm vascular conductance responses to sustained and rhythmic isometric exercise and arterial occlusion in trained rock climbers and untrained sedentary subjects. European Journal of Applied Physiology, 76, 174-180.

Gamble, J., Gartside, I. B., \& Christ, F. (1993). A reassessment of mercury in silastic strain gauge plethysmography for microvascular permeability assessment in man. Journal of Physiology, 464, 407-422.

Green, D. J., Maiorana, A., O’Driscoll, G., \& Taylor, R. (2004) Effect of exercise training on endothelium-derived nitric oxide function in humans. Journal of Physiology, 561(1), 1-25

Green, D. J., Spence, A., Rowley, N., Thijssen, D. H. J., \& Naylor, L. H. (2012). Vascular adaptation in athletes: is there an “athlete’s artery”? Experimental Physiology, 97(3), 295304.

Hambrecht, R., Adams, V., Erbs, S., Linke, A., Krankel, N., Shu, Y., Baither, Y., Geilen, S., Thiele, H., Gummert, J. F., Mohr, F. W., \& Schuler, G. (2003). Regular physical activity improves endothelial function in patients with coronary artery disease by increasing phosphorylation of endothelial nitric oxide synthase. Circulation, 107, 3152-3158.

Hoppeler, H. (1999). Vascular growth in hypoxic skeletal muscle. Advances in Experimental Medicine and Biology, 474, 277-286. 
Hunt, J. E. A., Walton, L. A., \& Ferguson, R. A. (2012). Brachial artery modifications to blood flow-restricted handgrip training and detraining. Journal of Applied Physiology, 112, 956-961.

Hunt, J. E. A., Galea, D., Tufft, G., Bunce, D., \& Ferguson, R. A. (2013) Time course of regional vascular adaptations to low load resistance training with blood flow restriction. Journal of Applied Physiology, 115, 403-411

Huonker, M., Schmid, A., Schmidt-Trucksass, A., Grathwohl, D., \& Keul, J. (2003). Size and blood flow of central and peripheral arteries in highly trained able-bodied and disabled athletes. Journal of Applied Physiology, 95, 685-691.

Kallman, D. A., Plato, C. C., \& Tobin, J. D. (1990) The role of muscle loss in the age-related decline of grip strength: cross-sectional and longitudinal perspectives. Journal of Gerontology, 45(3), M82-M88.

Kingwell, B. A., Sherrard, B., Jennings, G. L., \& Dart, A. M. (1997) Four weeks of cycle training increases basal production of nitric oxide from the forearm. American Journal of Physiology, 272(3), H1070-1077

Kumar, V. B., Viji, R. I., Kiran, M. S., \& Sudhakaran, P. R. (2007) Endothelial cell response to lactate: implication of PAR modification of VEGF. Journal of Cellular Physiology, 211(2), 477-485.

Langille B. L. \& O’Donnell, F. (1986). Reductions in arterial diameter produced by chronic decreases in blood flow are endothelium-dependent. Nature, 231, 405-407.

Laughlin, M. H., Newcomer, S. C., \& Bender, S. B. (2008). Importance of hemodynamic forces as signals for exercise-induced changes in endothelial cell phenotype. Journal of Applied Physiology, 104, 588-600. 
Lehmann, M., Schmid, P., \& Keul, J. (1984) Age- and exercise-related sympathetic activity in untrained volunteers, trained athletes and patients with impaired left-ventricular contractility. European Heart Journal, 5(suppl E), 1-7.

Maeda, S., Sugawara, J., Yoshizawa, M., Otsuki, T., Shimojo, N., Jesmin, S., Ajisaka, R., Miyauchi, T., \& Tanaka, H. (2009) Involvement of endothelin-1 in habitual exercise-inducted increase in arterial compliance. Acta Physiologica, 196(2), 223-229

Macleod, D., Sutherland, D. L., Buntin, L., Whitaker, A., Aitchison, T., Watt, I., Bradley, J., \& Grant, S. (2007). Physiological determinants of climbing-specific finger endurance and sport rock climbing performance. Journal of Sports Sciences, 25, 1433-1443.

Martin, W. H., Montgomery, J., Snell, P. G., Corbett, J. R., Sokolov, J. J., Buckey, J. C., Malony, D. A., \& Blomqvist, C. G. (1987). Cardiovascular adaptations to intense swim training in sedentary middle-aged men and women. Circulation, 75, 323-330.

Naylor, L. H., Weisbrod, C. J., O'Driscoll, G., \& Green, D. J. (2005). Measuring peripheral resistance and conduit arterial structure in humans using Doppler ultrasound. Journal of Applied Physiology, 98(6), 2311-2315.

Naylor, L. H., Fitzsimons, M. G., Cable, N. T., Thijssen, D. H. J., \& Green, D. J. (2011). Repeated increases in blood flow, independent of exercise, enhance conduit artery vasodilator function in humans. American Journal of Physiology. Heart and Circulation Physiology, 300(2), 664-669.

Newcomer, S. C., Thijssen, D. H. J. \& Green, D. J. (2011). Effects of exercise on endothelium and endothelium/smooth muscle cross talk: role of exercise-induced hemodynamics. Journal of Applied Physiology, 111, 311-320. 
Noẻ, F., Quaine, F., \& Martin, L. (2001) Influence of steep gradient supporting walls in rock climbing: biomechanical analysis. Gait and Posture, 13, 86-94.

Petersen, S. E., Wiesmann, F., Hudsmith, L. E., Robson, M. D., Francis, J. M., Selvanayagam, J. B., Neubauer, S., \& Channon, K. M. (2006). Functional and structural vascular remodeling in elite rowers assessed by cardiovascular magnetic resonance. Journal of the American College of Cardiology, 48, 790-797.

Prior, B. M., Yang, H. T., \& Terjung, R. L. (2004). What makes vessels grow with exercise training? Journal of Applied Physiology, 97, 1119-1128.

Rowley, N. J., Dawson, E. A., Birk, G. K., Cable, N. T., George, K., Whyte, G., Thijssen, D. H., \& Green, D. J. (2011a). Exercise and arterial adaptation in humans: uncoupling localized and systemic effects. Journal of Applied Physiology, 110, 1190-1195.

Rowley, N. J., Dawson, E. A., Hopman, M. T. E., George, K. P., Whyte, G., Thijssen, D. H. J., \& Green, D. J. (2011b) Conduit diameter and wall remodelling in elite athletes and spinal cord injury. Medicine and Science in Sports and Exercise, 44(5). 844-849.

Rudic, R. D., Shesely, E.G., Maedo, N., Smithies, O., Segal, S. S., \& Sessa, W. C. (1998). Direct evidence for the importance of endothelium-derived nitric oxide in vascular remodelling. Journal of Clinical Investigation, 101, 731-736.

Sadamoto, T., Bonde-Petersen, F., \& Suzuki, Y. (1983) Skeletal muscle tension, flow, pressure, and EMG during sustained isometric contractions in humans. European Journal of Applied Physiology, 51, 395-408.

Schmidt-Trucksäss, A., Schmid, A., Brunner, C., Scherer, N., Zäch, G., Keul, J., \& Huonker, M. (2000). Arterial properties of the carotid and femoral artery in endurance-trained and paraplegic subjects. Journal of Applied Physiology, 89, 1959-1963. 
Sjøgaard, G., Savard, G., \& Juel, C. (1988). Muscle blood flow during isometric activity and its relation to muscle fatigue. European journal of applied physiology and occupational physiology, 57(3), 327-335.

Sheel, A. W. (2004) Physiology of sport rock climbing. British Journal of Sports Medicine, 38(3), 355-359.

Sheel, A. W., Seddon, N., Knight, A., McKenzie, D. C., \& Warburton, D. E. (2003) Physiological responses to indoor rock-climbing and their relationship to maximal cycle ergometry. Medicine and Science in Sports and Exercise, 35(7), 1225-1231.

Sinoway, L. I., Musch, T. I., Minotti, J. R., \& Zelis, R. (1986). Enhanced maximal metabolic vasodilation in the dominant forearms of tennis players. Journal of Applied Physiology, 61, 673-678.

Snell, P. G., Martin, W. H., Buckey, J. C., \& Bloomqvist, C. G. (1987). Maximal vascular leg conductance in trained and untrained men. Journal of Applied Physiology, 62, 606-610.

Takano, H., Morita, T., Iida, H., Asada, K., Kato, M., Uno, K., Hirose, K., Matsumoto, A., Takenaka, K., Hirata, Y., Eto, F., Nagai, R., Sato., Y., \& Nakajima, T. (2005) Hemodynamic and hormonal responses to a short-term low-intensity resistance exercise with the reduction of muscle blood flow. European Journal of Applied Physiology, 95(1), 65-73.

Thijssen, D. H., Dawson, E. A., Black, M. A., Hopman, M. T., Cable, N. T., \& Green, D. J. (2008) Brachial artery blood flow responses to different modalities of lower limb exercise. Medicine \& Science in Sports and Exercise, 41(5), 1072-1079

Tinken, T. M., Thijssen, D. H. J., Black, M. A., Cable, T., \& Green, D. J. (2008). Time course of chance in vasodilator function in humans and capacity in response to exercise training in humans. Journal of Physiology, 586(20), 5003-5012. 
Tinken, T. M., Thijssen, D. H. J., Hopkins, N., Dawson, E. A., Cable, N. T. \& Green, D. J. (2010). Shear stress mediates endothelial adaptations to exercise training in humans. Hypertension, 55, 312-318.

Vallance, P., Collier, J., \& Moncada, S. (1989) Effects of endothelium-derived nitric oxide on peripheral arteriolar tone in man. The Lancet, 334(8670), 997-1000.

Watts, P. B., Martin, D. T., \& Durtschi, S. (1993). Anthropometric profiles of elite male and female competitive sport rock climbers. Journal of Sports Sciences, 11(2), 113-117.

Welsch, M. A., Blalock, P., Credeur, D. P., \& Parish, T. R. (2013) Comparison of brachial artery vasoreactivity in elite power athletes and aged-matched controls. PLoS ONE, 8(1), e54718

Zeppilli, P., Vanniculli, R., Dello Russo, A., Palmieri, V., Cameli, S., Corsetti, S., \& Pietrangeli, L. (1995). Echocardiographic size of conductance vessels in athletes and sedentary people. International Journal of Sports Medicine, 16, 38-44. 
Table 1. Anthropometric data of participants

\begin{tabular}{lccc}
\hline & CON & CLIMB & t-test \\
\hline Age (years) & $23 \pm 2$ & $24 \pm 3$ & 0.571 \\
Height (cm) & $175 \pm 7$ & $180 \pm 11$ & 0.260 \\
Body mass (kg) & $73 \pm 12$ & $72 \pm 8$ & 0.794 \\
BMI (kg.m ${ }^{2}$ ) & $23.8 \pm 2.6$ & $22.3 \pm 2.0$ & 0.197 \\
Systolic blood pressure (mmHg) & $129 \pm 15$ & $125 \pm 6$ & 0.548 \\
Diastolic blood pressure (mmHg) & $71 \pm 11$ & $67 \pm 2$ & 0.309 \\
Resting heart rate (bpm) & $60 \pm 10$ & $59 \pm 10$ & 0.844 \\
Forearm circumference (cm) & $25.6 \pm 2.1$ & $27.4 \pm 1.8$ & 0.082 \\
MVC (kg) & $37 \pm 9$ & $46 \pm 8$ & 0.055 \\
MVC / body mass & $0.50 \pm 0.11$ & $0.64 \pm 0.12$ & 0.032 \\
\hline BMI = body mass index; bpm = beats per minute; MVC = maximal voluntary contraction. \\
Values are mean \pm standard deviation
\end{tabular}


Table 2. Brachial artery characteristics in rock climbers and control participants

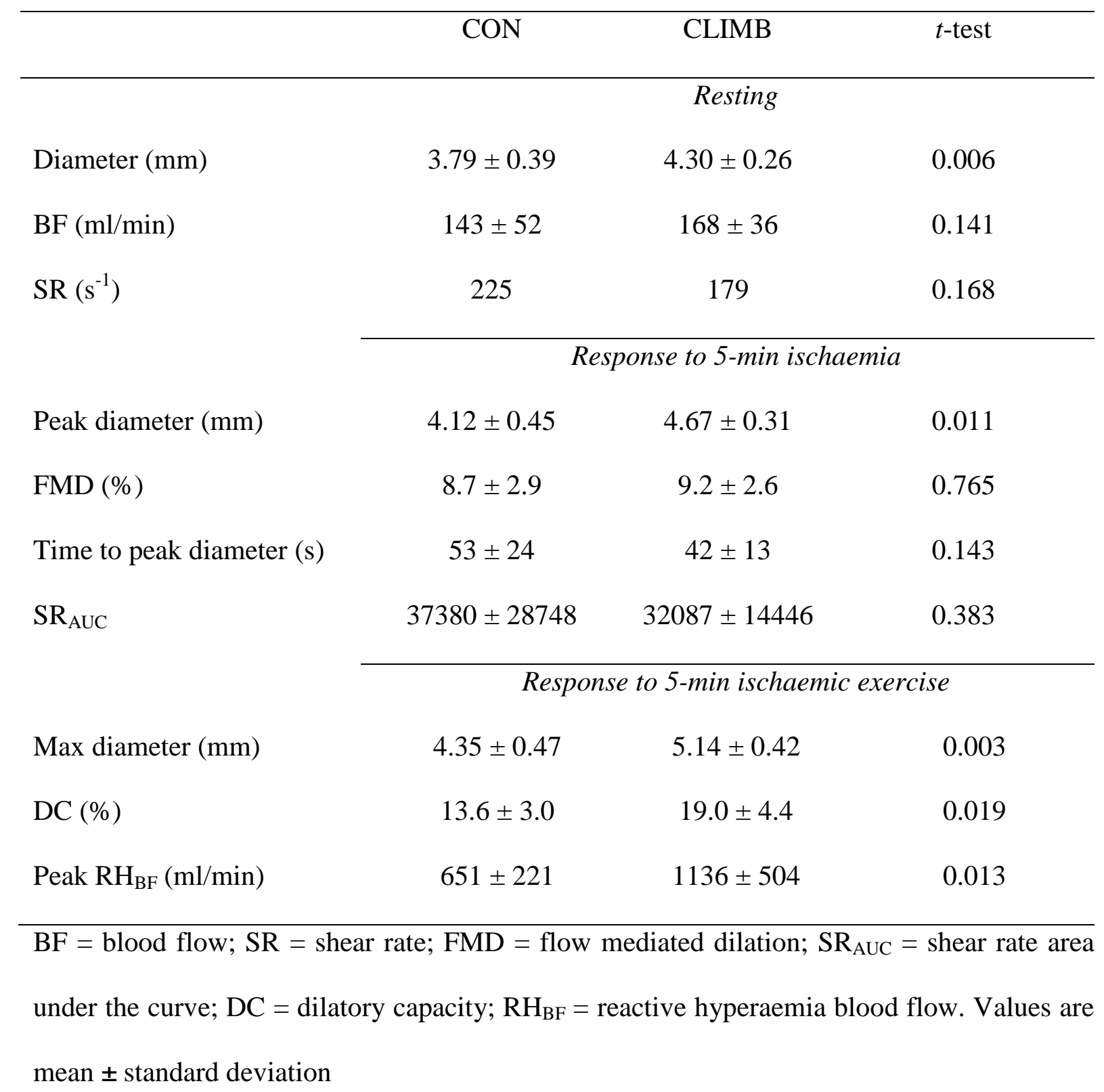


Table 3. Micro-vascular filtration capacity in rock climbers and control participants

\begin{tabular}{lccc}
\hline & CON & CLIMB & t-test \\
\hline Resting BF $\left(\mathrm{ml} .100 \mathrm{ml}^{-1} \cdot \mathrm{min}^{-1}\right)$ & $3.7 \pm 1.1$ & $3.6 \pm 1.8$ & 0.858 \\
$\mathrm{~K}_{\mathrm{f}}\left(\mathrm{ml} \cdot \mathrm{min}^{-1} \cdot \mathrm{mmHg}^{-1} .100 \mathrm{ml}\right.$ tissue & \\
& $3.8 \pm 0.9$ & $5.2 \pm 0.7$ & 0.010 \\
\hline $\mathrm{BF}$ = blood flow; $\mathrm{Kf}=$ capillary filtration. Values are mean \pm standard deviation &
\end{tabular}


Table 4. Correlations used to check size independence of brachial artery diameter

\begin{tabular}{|c|c|c|c|c|c|c|}
\hline & \multicolumn{2}{|c|}{ Resting diameter } & \multicolumn{2}{|c|}{ Peak diameter } & \multicolumn{2}{|c|}{ Max diameter } \\
\hline & $r$ value & $P$ value & $r$ value & $P$ value & $r$ value & $P$ value \\
\hline Diameter/BM & -0.062 & 0.819 & -0.089 & 0.743 & -0.152 & 0.587 \\
\hline Diameter/BM:BM & -0.802 & $<0.001$ & -0.803 & $<0.001$ & -0.755 & 0.001 \\
\hline Diameter/BM $^{b}: \mathrm{BM}$ & -0.422 & 0.103 & -0.451 & 0.079 & -0.466 & 0.080 \\
\hline Diameter/FC & 0.374 & 0.153 & 0.347 & 0.188 & 0.351 & 0.200 \\
\hline Diameter/FC:FC & -0.387 & 0.138 & -0.386 & 0.140 & -0.229 & 0.412 \\
\hline
\end{tabular}

$\mathrm{BM}=$ body mass; $\mathrm{FC}=$ forearm circumference 